\title{
EchoGéo
}

$57 \mid 2021$

Les enjeux de l'alimentation en eau potable des villes

\section{Le traitement médiatique des drogues dans la presse quotidienne française (2013-2018)}

Présentation d'une méthode d'analyse textuelle à l'aide du logiciel Iramuteq

Media treatment of drugs in the French daily press (2013-2018): presentation of a textual analysis method using Iramuteq software

Cécilia Comelli, Grégoire Le Campion et Marie Jauffret-Roustide

\section{(2) OpenEdition}

Journals

Édition électronique

URL : https://journals.openedition.org/echogeo/22277

DOI : $10.4000 /$ echogeo.22277

ISSN : 1963-1197

Éditeur

Pôle de recherche pour l'organisation et la diffusion de l'information géographique (CNRS UMR 8586)

Référence électronique

Cécilia Comelli, Grégoire Le Campion et Marie Jauffret-Roustide, « Le traitement médiatique des drogues dans la presse quotidienne française (2013-2018) », EchoGéo [En ligne], 57 | 2021, mis en ligne le 15 octobre 2021, consulté le 08 novembre 2021. URL : http://journals.openedition.org/ echogeo/22277; DOI : https://doi.org/10.4000/echogeo.22277

Ce document a été généré automatiquement le 8 novembre 2021.

EchoGéo est mis à disposition selon les termes de la licence Creative Commons Attribution - Pas d'Utilisation Commerciale - Pas de Modification 4.0 International (CC BY-NC-ND) 


\section{Le traitement médiatique des drogues dans la presse quotidienne française (2013-2018)}

Présentation d'une méthode d'analyse textuelle à l'aide du logiciel Iramuteq

Media treatment of drugs in the French daily press (2013-2018): presentation of a textual analysis method using Iramuteq software

Cécilia Comelli, Grégoire Le Campion et Marie Jauffret-Roustide

\section{Introduction}

1 Les discours médiatiques participent à façonner les représentations sociales, les représentations spatiales et les imaginaires géographiques. Ainsi, dans le cadre du projet Drusec ${ }^{1}$ qui s'intéresse aux enjeux de sécurité liés aux drogues, il a été décidé d'analyser le champ lexical des drogues (illégales) dans la presse quotidienne afin, d'une part, de tester une méthode encore peu explorée dans nos disciplines et qui allie des approches quantitatives et qualitatives, et, d'autre part, d'étudier les représentations véhiculées par ce type de discours. Cependant, il est important de rappeler que les discours médiatiques sur les drogues que nous présentons ici constituent un point de vue, qui reflète et influence en partie celui du grand public ou des politiques. Il en existe d'autres, celui des usagers, des professionnels ou des chercheurs que nous étudions par le biais d'autres méthodes au sein du projet mais qui ne sont pas l'objet de cet article.

\section{Contextes médiatique et socio-politique des drogues en France}

2 L'usage de drogues est un sujet fortement chargé émotionnellement qui donne lieu à la production de représentations sociales marquées par des stéréotypes moraux oscillant 
entre fascination et répulsion (Jauffret-Roustide, 2009). Cette prédominance de la morale dans les représentations sociales et spatiales des drogues en France est liée à la législation française qui est l'une des plus répressives d'Europe: l'usage simple de drogues pouvant être considéré comme un délit (Obradovic, 2015). Cette loi répressive a un impact sur les représentations sociales et tend à produire des figures caricaturales telles que celle du "drogué délinquant à punir » ou « du malade à soigner » (JauffretRoustide et Granier, 2017). Les médias participent à véhiculer ces discours et ces représentations. Ils contribuent à créer des "paniques morales» (Fredrickson et al. 2019), influencent les représentations socio-spatiales et les politiques des drogues, mais ils sont également le reflet de ces politiques (Bregman, 1989; Burgess, 1990; Beck \& Peretti-Watel, 2001, Lancaster et al., 2011).

3 L'étude de la presse s'est particulièrement développée depuis sa disponibilité via internet (Dagiral et Parasie, 2010) et le "tournant numérique » dont ont bénéficié les sciences sociales (Ash J. et al., 2016). En effet, la mise en ligne de la presse et son accès grâce à des bases de données telles que Factiva et Europresse, ces "réservoirs de données numériques» (Venturini, 2012), permettent une extraction facilitée de ces données, lesquelles peuvent être traitées aisément grâce à des outils informatiques. La sociologie, la linguistique ou la communication sont les disciplines phares dans l'utilisation des méthodes d'analyse textuelles, la géographe France Guérin-Pace semble être une des pionnières de sa discipline en France à les utiliser (Guérin-Pace, 1997). Par ailleurs, la géographie, bien que timidement, a aussi fait de la presse un objet d'étude. On peut ainsi citer les travaux des géographes anglo-saxons Burgess et Gold (1985), plus récemment Mains, Cupples et Lukinbeal (2015) et en France, la parution d'un double numéro de l'Espace géographique consacré à « Géographie et médias » qui : «[...] visait à interroger les espaces géographiques révélés par les contenus médiatiques" (Beauguitte et al., 2016), ou encore l'ANR Géomédia (2013-2016)². Finalement, la presse est une source d'information supplémentaire et complémentaire aux sources plus traditionnelles que sont l'entretien, le questionnaire ou l'observation afin d'étudier ce qui est devenu central dans la géographie sociale depuis les années 1970: les représentations (Frémont, 1976; Debarbieux, 1998; Di Méo, 2008). L'analyse textuelle automatisée est une des méthodes pour appréhender ces sources.

4 Les études portant sur les représentations sociales des drogues sont rares en France et produites essentiellement à partir d'approches en psychologie sociale (Dany et Apostolidis, 2002) ou d'enquêtes sur les opinions telles que l'enquête EROPP (enquête sur les Représentations, Opinions et Perceptions sur les Psychotropes) ${ }^{3}$. Plus rares encore sont les recherches menées sur l'analyse des drogues et des politiques à travers l'étude de la presse (Jauffret-Roustide et Cailbault, 2018). La sociologie et la psychologie sociale ont apporté beaucoup aux drug studies, cependant, la dimension spatiale des usages et des représentations de la drogue y est souvent absente. Quant aux travaux des géographes ayant pour objet central les drogues, ils ciblent surtout la dimension géopolitique ${ }^{4}$.

\section{Contexte de l'étude : le projet Drusec et la géographie des drogues}

Notre étude s'inscrit dans le projet de recherche franco-allemand Drusec qui questionne les pratiques, les représentations et les politiques urbaines liées à l'usage de produits psychoactifs à travers différentes méthodes cartographiques et des sciences 
humaines. Afin d'aborder la question sous des angles complémentaires et de prendre en considération l'ensemble des parties prenantes, la recherche a été menée auprès d'habitant.es (consommateurs ou non), d'usageres et de professionnelles des centres d'accompagnement médico-sociaux et de représentant.es de l'État, via des entretiens ou par la réalisation de cartes sensibles.

6 Pour compléter ces approches, nous avons choisi d'étudier un corpus de textes homogènes (la presse quotidienne) et thématique (sur les drogues) (Cabré, 2008). Ainsi, la manière dont la presse reflète les représentations sociales et spatiales des drogues sur la période 2013-2018 a été étudiée à partir d'une approche textométrique. C'est-àdire à partir d'un traitement statistique du vocabulaire utilisé dans les articles de presse traitant des drogues. "L'évolution de désignation de la «lexicométrie » en «textométrie » veut exprimer que l'analyse menée ne se cantonne pas à l'étude du lexique, mais investit l'ensemble du texte » (Pincemin, 2011). Parmi ces médias, nous nous sommes intéressés à la presse écrite nationale $(\mathrm{PQN})$ et régionale $(\mathrm{PQR})$. Pour la $\mathrm{PQR}$, nous avons ciblé des titres de la région Nouvelle Aquitaine qui est le principal terrain de l'équipe française du projet Drusec.

$7 \quad$ Notre approche nous semble novatrice tant par le choix de la méthode employée qui est encore peu répandue en géographie et dans les drug studies (l'analyse textométrique de la presse) que par l'objet de cette étude (les dimensions géographiques des drogues). La pertinence de cette méthode exploratoire en sciences sociales a cependant été démontrée par F. Guérin-Pace dès 1997, qui l'a appliquée dans un article co-écrit présentant les orientations prises par la revue l'Espace géographique par l'analyse des titres et des mots-clés à l'occasion des 40 ans de la revue (Guérin-Pace et al., 2012).

L'objectif de notre analyse est d'interroger la manière dont la presse quotidienne traite de la question des drogues: quels sont les angles thématiques choisis d'une part, et, d'autre part, quelle(s) géographie(s) des drogues peut-on repérer à partir de ce traitement médiatique? Par géographie des drogues, nous entendons la spatialisation des pratiques et des politiques en lien avec les drogues ainsi que les représentations des lieux où se déroulent ces pratiques.

9 La première partie de l'article sera consacrée à la présentation de la méthode employée. Nous exposerons tout d'abord nos choix méthodologiques et décrirons les analyses textométriques menées. Nous présenterons ensuite nos résultats en deux temps : la dimension thématique puis la dimension spatiale. Nous montrerons ainsi que la presse est l'écho de tendances sociétales qui peuvent être présentées de manières différentes selon l'échelon de diffusion mais qu'elle révèle aussi une spatialisation des drogues à travers trois types de lieux à trois échelons : des noms de villes, des lieux génériques et des lieux spécifiques. Nous verrons ainsi comment le traitement médiatique des usages des drogues sur certains territoires, à travers l'étude des champs lexicaux et des entités nommées spatiales (Ehermann, 2008), participent à construire et à véhiculer des représentations et à la marginalisation de certains espaces et de certains individus.

\section{Les statistiques textuelles pour l'étude de la presse}

Pour notre étude des drogues dans la presse, nous avons opté pour une analyse statistique textuelle en faisant des articles de presse sélectionnés notre corpus. La méthodologie mise en œuvre pour l'analyse de la presse s'est déroulée en plusieurs 
temps, en suivant le processus décrit par T. Cabré, qui va de la constitution du corpus à l'utilisation d'outils de traitement automatisé (Cabré, 2008). La méthodologie est une proposition de l'équipe française, transmise à l'équipe allemande du projet pour sa mise en œuvre outre-Rhin. Un premier temps a consisté en la sélection du choix des titres de presse et des mots-clés. Pour avoir des résultats comparables à terme, le choix du corpus a été construit et validé par les équipes franco-allemandes du projet. Le deuxième temps a consisté à l'extraction des articles à analyser qui forment le corpus de textes via la base de données Europresse. Enfin, nous avons procédé en un traitement des données, à l'aide du logiciel Iramuteq ${ }^{5}$ qui s'appuie sur la méthode Alceste, du nom d'un autre logiciel d'analyse textuel conçu par le chercheur Max Reinert dans les années $1980^{6}$ (Reinert, 1990). Cette méthode, pour reprendre les mots de Max Reinert (1999) cherche à découper les textes en plusieurs parties ( «segments») de même étendue («longueur ») afin qu'ils soient comparables et à en étudier la structure à travers des méthodes d'analyse de distribution statistique des termes. L'objectif étant de faire ressortir des « classes de discours » (Fallery et Rhodain, 2007).

\section{La constitution du corpus}

11 Pour la préparation de la base de données, les journaux étudiés ont été sélectionnés selon trois critères: l'importance du tirage, la diversité des orientations éditoriales (pour la presse quotidienne nationale) et la diffusion sur les terrains d'étude réalisés dans le Sud-Ouest de la France (pour la presse quotidienne régionale) (voir tab.1). Parallèlement, une liste de mots appartenant au champ lexical de la drogue a été constituée et a servi à l'extraction des articles. Cette liste qui compose le champ lexical des pratiques liées à la consommation de produits stupéfiants se compose comme suit : - des noms génériques désignant des produits (ex.: drogue, produit psychoactif, traitement de substitution, etc.) ;

- des noms spécifiques de produits classés comme stupéfiants (ex. : cocaïne, cannabis, crack, etc.);

- des noms de lieux de consommation (salle de consommation à moindre risque, salle d'injection, salle de shoot);

- des pratiques (réduction des risques).

Une liste de mots à exclure du corpus, comportant certains termes qui ne correspondaient pas à notre recherche comme des noms de pays étranger ou le vocabulaire du sport (hippique notamment) a également été créée, pour éviter les articles qui traitent du « crack de la 5ème course » par exemple. Nous avons également exclu de la liste le mot " opioïde ", car les articles qui traitaient de la crise des opioïdes aux États-Unis étaient nombreux sur la période étudiée et parasitaient notre recherche sur la géographie de la drogue en France.

Nous avons choisi d'étudier la presse sur une période de cinq ans qui est une durée assez longue pour avoir un nombre suffisant d'articles pour rendre l'étude pertinente et en même temps assez restreinte pour faire émerger une certaine tendance quant $\mathrm{au}(\mathrm{x})$ discours émis et saisir une vision d'actualité de la question. Nous avons donc pris les cinq années qui précédaient la date du début de l'étude (mars 2018). Au total, près de 10000 articles ont été extraits et analysés (tableau 1). 
Tableau 1 - Récapitulatif du nombre d'articles extraits par titre

\begin{tabular}{|l|l|l|l|l|}
\hline \multicolumn{2}{|l|}{ Titre } & Tirage (2018) & Nombre d'articles extraits & Totaux \\
\hline \multirow{4}{*}{ LQ Libération } & 70000 & 649 & \\
\cline { 2 - 4 } & La Croix & 97000 & 493 & \multirow{2}{*}{5396} \\
\cline { 2 - 4 } & Le Monde & 302000 & 1086 & \\
\cline { 2 - 4 } & Le Figaro & 317000 & 1498 & \multirow{2}{*}{4564} \\
\cline { 2 - 4 } & 20 minutes & 524000 & 1670 & \\
\hline \multirow{2}{*}{ PQR } & Sud-ouest & 224000 & 3388 & \multirow{2}{*}{960} \\
\cline { 2 - 4 } & La Charente libre & 28000 & 1176 & \\
\hline
\end{tabular}

14 Les articles extraits dans leur version numérique, journaux par journaux, par groupe de 1000 au maximum, au format HTML, ont ensuite été rassemblés en un seul fichier CSV (tableur) afin de les organiser selon les variables suivantes: source, date, titre et auteur. Ce fichier a été converti au format TXT pour être importé dans le logiciel libre Iramuteq ${ }^{7}$. Ce logiciel, qui s'appuie sur le logiciel $\mathrm{R}^{8}$ et le langage Python, a été choisi car il permet de traiter un corpus contenant un grand nombre de textes pour réaliser des nuages de mots, des analyses de similitude ou des classifications hiérarchiques descendantes en utilisant la méthode Alceste. Ces analyses seront expliquées dans la partie suivante.

15 Avant de pouvoir commencer les analyses avec Iramuteq, il a fallu préparer le corpus et apporter quelques modifications au dictionnaire contenu dans le logiciel. Ainsi le corpus a été lemmatisé, c'est-à-dire que les verbes conjugués ont été ramenés à leur infinitif, les noms au singulier et les adjectifs au masculin singulier. De plus, le dictionnaire a été paramétré afin qu'il reconnaisse des expressions telles que garde à vue comme un tout et non comme trois formes différentes ou que le mot héroïne ne devienne pas héros par exemple lors de la lemmatisation. Le choix de la lemmatisation du corpus permet de regrouper des mots de la même famille et de réduire le nombre de formes analysées d'environ un quart (tableau 2). S'il faut rester attentif aux regroupements de formes qui ne seraient pas de la même famille, réduire le nombre de formes analysées permet de gagner du temps voire d'éviter que le matériel informatique «plante ».

Tableau 2 - Statistiques textuelles du corpus

\begin{tabular}{|l|l|l|l|l|}
\hline Presse & $\begin{array}{l}\text { Nombre de } \\
\text { textes }\end{array}$ & $\begin{array}{l}\text { Occurrences } \\
\text { (nombre total de } \\
\text { mots) }\end{array}$ & $\begin{array}{l}\text { Formes } \\
\text { (nombre de mots } \\
\text { différents) }\end{array}$ & $\begin{array}{l}\text { Formes } \\
\text { lemmatisées }\end{array}$ \\
\hline PQN & 5396 & 2884731 & 79797 & 56203 \\
\hline
\end{tabular}




\begin{tabular}{|l|l|l|l|l|}
\hline $\mathrm{PQR}$ & 4564 & 1840374 & 58653 & 41180 \\
\hline total & 9960 & 4725105 & 101204 & 74005 \\
\hline
\end{tabular}

Les analyses ont pu être menées sur le corpus complet et sur les sous-corpus regroupant d'une part la presse à diffusion nationale et, d'autre part, la presse à diffusion régionale. Les dimensions thématiques et spatiales des articles ont été explorées afin d'identifier les similitudes et les différences selon l'échelon de diffusion.

\section{Description des analyses réalisées}

17 La première requête demandée à Iramuteq a été la réalisation d'un nuage de mots. Ce nuage de mots présenté dans la partie suivante de l'article (voir illustration 1) permet d'avoir une première vision d'ensemble du corpus. Le graphique produit par le logiciel est constitué des formes lemmatisées dont la fréquence est la plus élevée dans le corpus. Plus ces formes apparaissent en gros, plus elles sont présentes dans l'ensemble du corpus. Dans le cas présent, les formes sélectionnées pour réaliser le nuage apparaissent plus de 900 fois chacune. Ce seuil de 900 occurrences et plus a été choisi après plusieurs essais car il permet une bonne lisibilité du graphique. Un seuil plus élevé (1 200 occurrences par exemple), réduit considérablement le nombre de formes affiché et le nuage est appauvri, à l'inverse, un seuil trop bas (500 fois et plus par exemple) rend le nuage illisible car trop fourni.

Ce nuage de mots permet de répondre rapidement aux premières questions qui se posent pour l'analyse de tout document, y compris pour un corpus de presse: de qui est-il question? de quoi parle-t-on? comment en parle-t-on? et de quel(s) lieu(x) s'agit-il ? Visuellement, le lecteur du graphique peut rapidement répondre à chacune de ces questions préliminaires et avoir une première idée du traitement médiatique de la question étudiée, ici les drogues dans la presse quotidienne française.

Dans un deuxième temps, nous avons réalisé une analyse textométrique s'appuyant sur un traitement statistique de la répartition du vocabulaire afin de faire émerger du corpus les "univers lexicaux» les plus représentatifs (Rouré et Reinert, 1993). La méthode Alceste s'appuie ensuite sur une classification hiérarchique descendante ${ }^{9}$ qui " cherche à rendre compte de l'organisation interne d'un discours " (Marpsat, 2010) en analysant les cooccurrences, c'est-à-dire les formes présentes simultanément, dans les différents segments de texte. Nous avons sélectionné différents paramètres du logiciel pour réaliser cette classification. Ces paramètres sont principalement :

- Le type grammatical des formes analysées : nous avons choisi d'analyser les formes actives, c'est-à-dire les adjectifs, les verbes, les adverbes, les noms communs et les formes non reconnues.

- Le nombre de classes à partir duquel découper le texte pour obtenir la classification optimale : ici, nous sommes partis de 10 classes (pour une classification optimale finale à 5 classes).

- Le nombre de formes maximales analysées: l'analyse n'a pas été menée sur tout le corpus mais sur les 5000 formes les plus présentes, ce qui est suffisamment représentatif et techniquement réalisable avec la capacité de traitement de nos ordinateurs. 
20 Chaque classe proposée suite à cette analyse regroupe les formes les plus souvent utilisées ensemble (co-occurence). Ainsi le contenu de chaque classe est homogène et en même temps différent des autres classes. Cette analyse a été réalisée séparément sur les sous-corpus de la $\mathrm{PQN}$ et de la $\mathrm{PQR}$ afin de pouvoir comparer ces dernières pour voir émerger des choix éditoriaux selon l'aire de diffusion. Le résultat graphique apparait sous la forme d'un dendrogramme qui représente la partition du corpus en classes et une indication de la taille de celles-ci par rapport à l'ensemble du corpus (illustrations 3 et 4).

21 Le logiciel permet également de consulter les métadonnées et les statistiques du corpus (illustration 2) pour connaître la liste de toutes les formes présentes, la fréquence d'apparition de chacune ou de connaitre son contexte d'utilisation en accédant aux segments de texte où elle apparait. Nous nous y référons régulièrement au cours de l'analyse, tout comme nous avons recours à l'analyse des spécificités qui permet d'obtenir la fréquence absolue et relative de chaque forme dans les différentes variables, ici dans chaque journal.

\section{Résultats : la presse, reflet de la politique et sensationnalisme}

22 L'analyse du traitement des données nous a permis, dans un premier temps, de faire ressortir les thématiques les plus développées dans la presse puis, dans un second temps, de localiser les faits relatés et enfin, d'étudier les représentations véhiculées de ces espaces. Iramuteq a également permis d'étudier séparément les presses quotidiennes nationale et régionale et de constater des nuances selon le type de presse sur ces deux aspects (thématiques et spatiaux).

\section{Les drogues dans la presse : le cannabis et un traitement avant tout judiciaire}

La lecture du nuage permet de voir que les acteurs mis en avant dans les articles sont des hommes plus souvent que des femmes, des jeunes, des policiers (ainsi que des enquêteurs et des gendarmes), des avocats (des juges et des procureurs), des personnes présentées comme des victimes (dont des morts), des trafiquants (parfois nommés dealers) et dans une moindre mesure des consommateurs. 


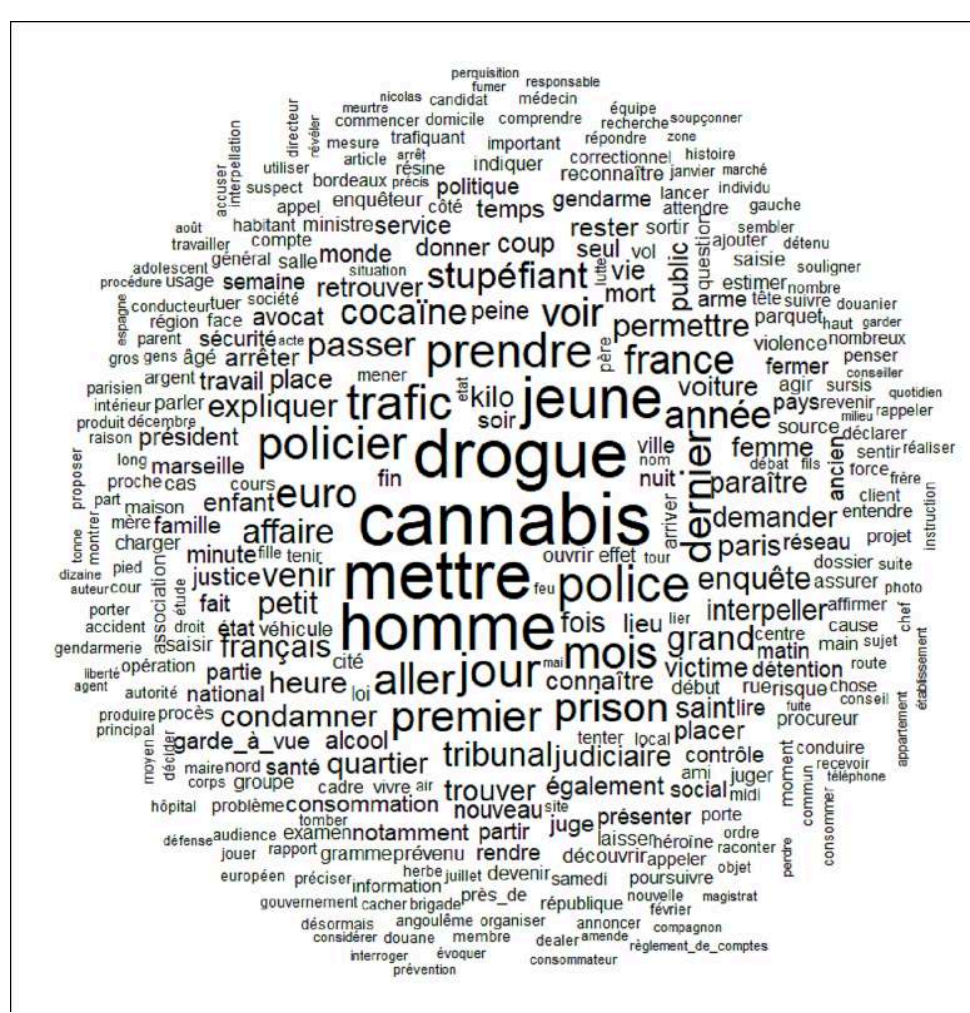

Dans un second temps, parmi les mots clés que nous avions renseignés, nous constatons sans surprise que les termes génériques de drogue et de stupéfiant sont bien présents mais notons également que les produits psychoactifs illicites dont il est le plus souvent question sont principalement le cannabis suivi par la cocaïne. Il est à noter que le terme alcool est cité fréquemment alors qu'il ne faisait pas partie de la liste des mots à rechercher. Ces trois produits licites et illicites sont les plus consommés (Beck et al., 2015), voire les mieux acceptés ou les moins tabous, ainsi que le révèlent entre autre l'enquête EROPP ou les travaux de Dany et Apostolidis (2002).

La présence de l'alcool peut s'expliquer pour plusieurs raisons. Tout d'abord, il s'agit d'un produit licite largement consommé par la population générale, ensuite la polyconsommation de produits psychoactifs est quasi-systématique chez les usagers dépendants et, enfin, l'alcool est très présent dans les contextes de violences que l'on peut retrouver dans ces articles, comme nous le verrons plus en détail.

Concernant la forte présence du cannabis dans la presse, elle est également liée au contexte politique. En effet, si le débat sur la légalisation revient régulièrement depuis 30 ans, en période pré-électorale en particulier, cette question a été mise à l'agenda politique ces derniers mois pour aboutir à une modification de la loi rendant l'usage de cannabis passible d'une amende forfaitaire délictuelle depuis le $1^{\mathrm{er}}$ septembre 2020 . Le débat récent sur l'usage du cannabis à visée thérapeutique ${ }^{10}$ est aussi une raison expliquant que ce soit le produit le plus mobilisé dans la presse. Le mouvement international de développement de la légalisation dans des pays de plus en plus nombreux à partir de 2012 a également contribué à mobiliser l'agenda politique sur les questions de cannabis, ce qui a donné lieu à des prises de position médiatiques de plus en plus nombreuses en France. 

dont la question des drogues est traitée par les journalistes. Parmi ces formes, nous retrouvons celles de trafic, police, prison, tribunal, condamner, enquête, interpeller ou judiciaire qui renvoient à la criminalisation inhérente à la législation française. Pour connaitre la fréquence exacte d'un mot dans le corpus, il est possible de consulter dans les statistiques du corpus la liste des formes actives prises en compte par le logiciel pour réaliser les différentes analyses (illustration 2).

Illustration 2 - Formes analysées pour la réalisation du nuage de mots

\begin{tabular}{|c|c|c|c|c|}
\hline Résumé & Formes actives $x$ & Formes supplémentaires & Total & Hapax \\
\hline Forme & & Freq. 8 & Types & \\
\hline cannabis & & 10382 & nom & \\
\hline drogue & & 9637 & nom & \\
\hline mettre & & 9240 & ver & \\
\hline homme & & 9042 & nom & \\
\hline jeune & & 7946 & adj & \\
\hline police & & 6776 & nom & \\
\hline prendre & & 6535 & ver & \\
\hline trafic & & 6479 & nom & \\
\hline jour & & 6430 & nom & \\
\hline policier & & 6093 & nom & \\
\hline mois & & 5924 & nom & \\
\hline aller & & 5861 & ver & \\
\hline premier & & 5727 & adj & \\
\hline prison & & 5598 & nom & \\
\hline dernier & & 5528 & adj & \\
\hline euro & & 5396 & nom & \\
\hline france & & 5246 & $\mathrm{nr}$ & \\
\hline voir & & 5142 & ver & \\
\hline année & & 4980 & nom & \\
\hline stupéfian & & 4614 & adj & \\
\hline cocaine & & 4567 & nom & \\
\hline passer & & 4503 & ver & \\
\hline affaire & & 4283 & nom & \\
\hline venir & & 4221 & ver & \\
\hline grand & & 4150 & adj & \\
\hline sudouest & & 4041 & $\mathrm{nr}$ & \\
\hline
\end{tabular}

Source : extrait d'Iramuteq.

Ainsi, par exemple, dans l'ensemble du corpus qui regroupe la $\mathrm{PQR}$ et la $\mathrm{PQN}$ dont est issu le nuage de mots réalisé, le mot cannabis est la forme active la plus fréquente avec plus de 10000 occurrences, homme arrive en $4{ }^{\text {ème }}$ place des formes actives avec plus de 9000 occurrences, police arrive en 6ème position avec 6776 occurrences, trafic est $8^{\text {ème }}$ avec 6479 occurrences et prison $14^{\text {ème }}$ avec 5598 occurrences.

L'étude du nuage de mots réalisé avec Iramuteq met ainsi en évidence un univers masculin, la prédominance du cannabis comme produit et un traitement judiciaire de la question des drogues dans la presse. Cette prédominance du judiciaire s'explique en partie par le choix politique français d'un cadre prohibitionniste qui criminalise la production, la possession, l'usage et la vente des produits classés comme stupéfiants et considère l'usage de drogues comme un délit. Une autre explication est liée à la tendance de la presse à s'inscrire dans une tonalité sensationnaliste, à laquelle se prête parfaitement le choix du judiciaire. En revanche, le nuage de mots ainsi que la liste des formes les plus présentes qui le compose ne montrent pas de spécificités géographiques 
flagrantes. Celles-ci, ainsi que des nuances thématiques, sont plus visibles via l'étude séparée des sous-corpus de la $\mathrm{PQN}$ et de la $\mathrm{PQR}$ avec la méthode Alceste.

\section{Des degrés de violence différents}

31 L'étape suivante pour la comparaison des sous-corpus PQN / PQR a été d'appliquer la méthode Alceste d'analyse textométrique, en sélectionnant les paramètres présentés dans la méthodologie (voir supra). Nous constatons que les classifications opérées sur les deux sous-corpus sont assez proches mais permettent de repérer des nuances dans le traitement de l'information. Les illustrations 3 et 4 sont les dendrogrammes des classifications extraits d'Iramuteq. On peut y voir pour chacune des classes, les termes les plus représentatifs.

Ainsi, dans les deux cas ( $P Q N$ et $P Q R)$, la classification a opéré en une première séparation des corpus en deux avec, d'une part, les univers lexicaux du trafic, du judiciaire et de la violence et, d'autre part, ceux des risques sanitaires, du politique et de la vie sociale. Ces deux classes principales ont ensuite de nouveau été divisées (une fois pour l'une, deux fois pour l'autre) pour obtenir cinq classes finales dont «les énoncés [...] sont similaires entre eux, et aussi différents que possible des énoncés d'une autre classe » (Marpsat, 2010). Plus les formes apparaissent en haut de la liste au sein d'une classe et en gros caractère, plus elles appartiennent significativement à cette classe.

Illustration 3 - Classification du corpus issu de la presse nationale

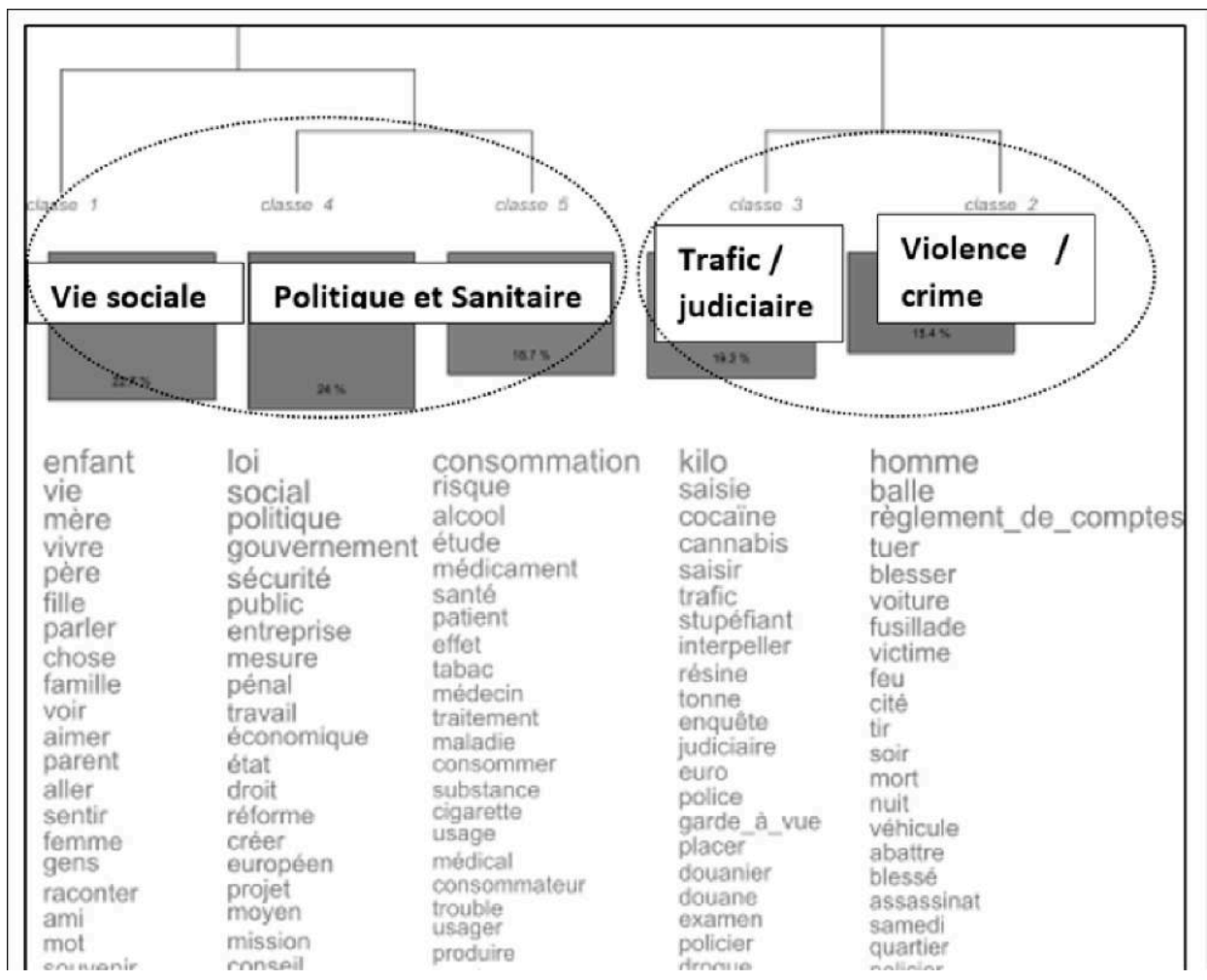

Source : dendrogramme extrait d'Iramuteq. 
Illustration 4 - Classification du corpus issu de la presse régionale

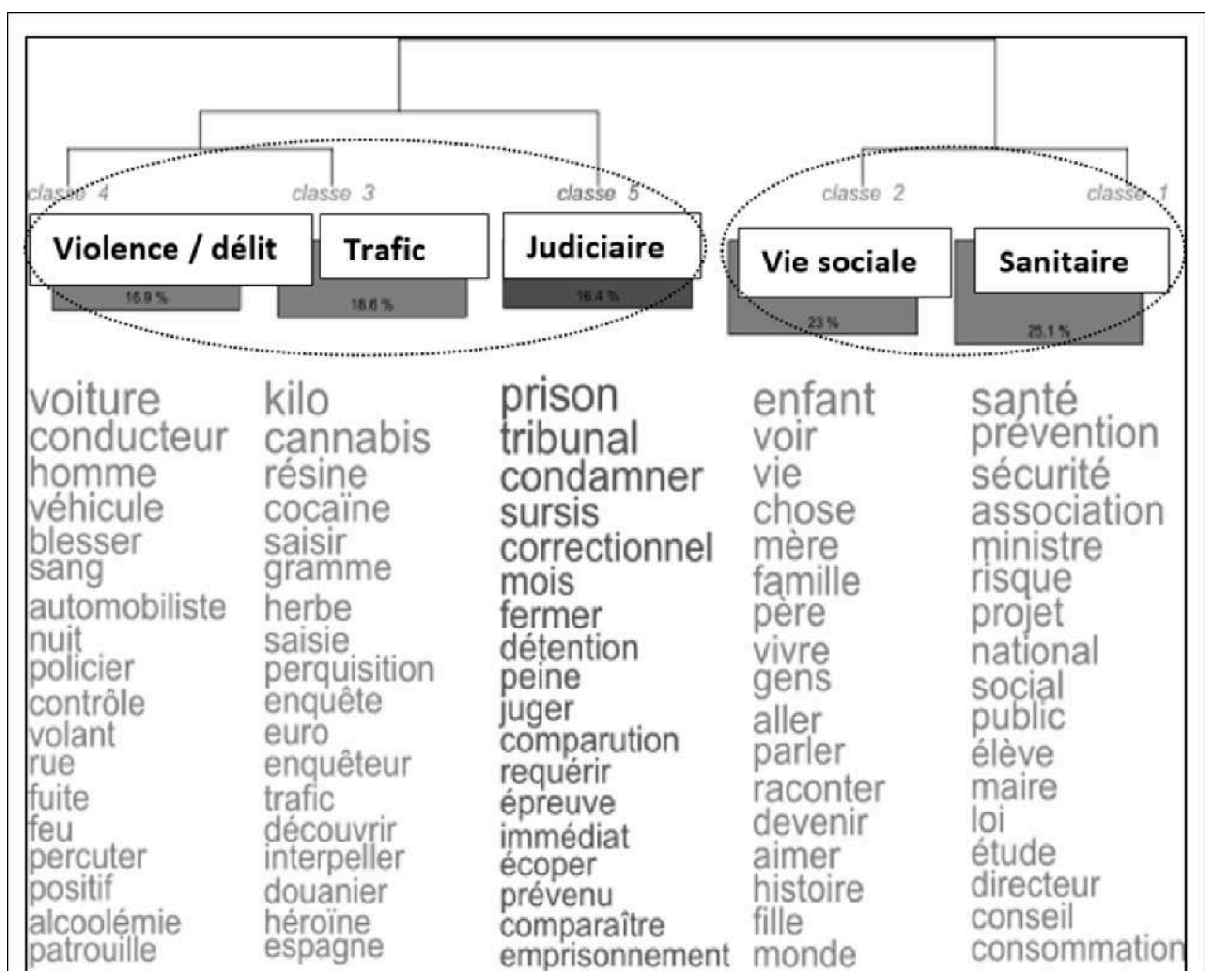

Source : dendrogramme extrait d'Iramuteq

\section{Drogue et violence : crimes vs délits}

Les grandes thématiques qui ressortent des classifications opérées sur les presses nationale et régionale sont proches, cependant les nuances observées révèlent un focus différent de l'information selon le type de presse. Nous ne présentons pas ici les différences qu'il peut exister au sein de chaque journal, en fonction de leurs lignes éditoriales notamment, pour nous concentrer sur la comparaison PQN / PQR. Dans le cas de la presse quotidienne nationale (illustration 3), la catégorie que l'on a choisi de nommer « violence $~^{11}$ relate des faits bien plus violents, relevant plus du crime que du délit que dans la classe similaire de la presse régionale ${ }^{12}$. Dans cette $P Q N$, il est clairement question de morts violentes ainsi que le montrent les extraits d'articles présentés ci-après et les mots visibles parmi la vingtaine présente sur le graphique tels que : tuer, mort, homicide, assassinat, abattre ou règlement de compte. Dans le détail, ces décès sont dus à des fusillades, des coups de feu, des tirs avec des balles et ont lieu notamment le soir ou la nuit du samedi dans des cités ou des quartiers. Depuis le tableau statistique (tableau 2), il est également possible d'avoir accès aux contextes d'utilisation d'une forme (en cliquant dessus) afin de confirmer les hypothèses émises à partir de la classification (voir les extraits cités par la suite). Ainsi, cette seule catégorie révèle la mise en avant d'un imaginaire stéréotypé autour de la drogue et de la violence dans les grands ensembles périphériques, nommés cités ou quartiers dans la presse (Sedel, 2009). Les faits relatés par ces journaux sont bien réels, mais c'est un choix éditorial de les traiter d'une part, et de le faire sous un angle sensationnel de l'autre. Véhiculer ces faits divers participent à renforcer l'image négative (Derville, 1997) des usagers et des lieux de vie auxquels ils sont parfois assignés. 
Extraits de la $\mathrm{PQN}$ à partir de la catégorie « violence »

Quinze personnes doivent être présentées à la justice aujourd'hui et demain après une importante saisie d'armes et de drogues dans une cité de Marseille a-t-on appris de source policière. (Le Figaro, 13/10/2016)

Un homme de 24 ans a été tué de plusieurs balles dans la nuit de mercredi à jeudi à Marseille lors d'un probable nouveau règlement de comptes dans les quartiers nord dont les habitants se sont récemment fédérés pour dénoncer la violence. (Libération, 20/06/2013)

La violence routinière continue à Marseille jeudi soir deux hommes ont été tués dans une voiture retrouvée criblée de balles sur le parking d'un centre commercial dans l'est de la ville a-t-on appris de source policière. (Libération, 24/09/2013)

Les articles de la presse régionale analysés traitent également des faits divers et de violence, mais cette catégorie semble aborder des faits moins violents que ne le fait la $P Q N$. Il n'y est pas d'emblée question de décès (dont il est aussi question lors de l'analyse fine de cette catégorie mais de manière moins remarquable), ce sont surtout les délits routiers liés à l'alcool qui sont mis en avant. Cependant, si ces articles ont été sélectionnés lors de la constitution du corpus, c'est qu'ils contiennent au moins un des mots-clés du champ lexical de la drogue dont ni violence ni alcool ne font partie. Par ailleurs, les études sur les drogues, notamment celles menées par l'Observatoire français des drogues et des toxicomanies (OFDT), montrent que la poly-consommation est fréquente chez les usagers de produits psychoactifs et en particulier l'association avec l'alcool (Brissot et al., 2019). Ainsi, on retrouve cette présence conjointe de produits psychoactifs (stupéfiants et alcool) dans les articles, qu'il s'agisse de consommation ou de détention.

Extraits de la $\mathrm{PQR}$ à partir de la catégorie « violence »

Angoulême. Vers 3 heures un conducteur a tenté d'échapper à un contrôle avant d'être intercepté rue de Bordeaux. Âgé de 31 ans, il a été contrôlé positif aussi bien pour la prise d'alcool 0,9 g/l que de stupéfiants. (Charente-Libre, 09/12/2017)

Lors d'un contrôle d'alcoolémie mené route de Bordeaux, la police arrête un conducteur qui roulait avec $0,74 \mathrm{~g}$ d'alcool dans le sang, il a écopé d'une contravention. (Sud-Ouest, 19/03/2017)

L'étude de cette catégorie permet de montrer que la PQN et $\mathrm{PQR}$ abordent les faits mis en avant sur deux registres différents en termes de niveaux et de types de violence. On constate une tendance plus sensationnaliste dans la presse nationale alors que la presse régionale se focalise plus sur des faits divers locaux.

\section{Le trafic : grammes de cannabis en région vs tonne de produits dans la presse nationale}

Parmi les autres choix éditoriaux, la thématique du trafic ressort. D'après le vocabulaire utilisé, les deux échelons de diffusion abordent le trafic de manière assez similaire: dans les deux cas, les articles sont composés d'un vocabulaire qui fait référence au processus de criminalisation inhérent à la politique française en matière de drogue: saisie, interpellation, enquête, etc. La presse régionale semble cependant traiter de plus petits trafics que la presse nationale. En effet, les formes co-occurrentes de trafic dans la $\mathrm{PQR}$ sont plus souvent le cannabis, sous forme de résine et d'herbe, et si le kilo est l'unité de mesure la plus utilisée, il est ici régulièrement question de gramme, ce qui est beaucoup plus rare dans la $\mathrm{PQN}$ où l'unité de la tonne est plus utilisée concernant le trafic (tableau 3) et où la cocaïne arrive avant le cannabis. La fréquence d'apparition plus importante de certaines formes est pertinente pour l'analyse et celle- 
ci est enrichie par l'étude de la coprésence des formes, ce que permet de faire la classification notamment. L'analyse des spécificités qui donne accès aux effectifs relatifs de chaque forme dans les différentes sources du corpus permet de confirmer les résultats.

Tableau 3 - Les co-occurrences liées au trafic, dans les presses nationale et régionale

\begin{tabular}{|l|l|l|l|l|}
\hline \multirow{2}{*}{ Formes } & \multicolumn{2}{l|}{$\begin{array}{l}\text { Occurrences } \\
\text { PQN }\end{array}$} & \multicolumn{2}{l|}{$\begin{array}{l}\text { Occurrences } \\
\text { PQR }\end{array}$} \\
\cline { 2 - 5 } & Val. Abs & $\%$ & Val. Abs & $\%$ \\
\hline Résine & 674 & 1,2 & 1142 & 2,8 \\
\hline Herbe & 390 & 0,7 & 828 & 2 \\
\hline Cocaïne & 2629 & 4,7 & 1938 & 4,7 \\
\hline Gramme & 492 & 0,9 & 1480 & 3,6 \\
\hline Tonne & 648 & 1,2 & 333 & 0,8 \\
\hline
\end{tabular}

Deux hypothèses peuvent expliquer les différences entre presse nationale et presse régionale. Premièrement, il est de la vocation de la $\mathrm{PQR}$ de proposer une information spatialisée dans sa zone de diffusion (Ballarini, 2008). Or, les faits de petite délinquance, tels que les délits routiers et les saisies de quelques grammes de cannabis sont plus fréquents au quotidien que ceux relevant du grand banditisme et n'intéressent guère les lecteurs au-delà du territoire où ils ont eu lieu, d'où leur absence dans la PQN. D'autre part, les titres de la presse régionale choisis n'ont pas de concurrence dans leurs aires de diffusion (Albert, 2016), à la différence de la PQN qui offre une diversité de choix aux lecteurs. Dans un contexte où la presse, et particulièrement la PQN, rencontre des difficultés (Delporte et al., 2016), cette dernière a peut-être plus la nécessité d'accrocher le lectorat, et ce en traitant de faits sensationnels, la rubrique des faits divers étant particulièrement lue (Leteinturier, 2021).

Outre les thématiques abordées et la manière dont elles le sont selon l'échelon de diffusion, l'étude de la presse a également permis de relever une autre géographie des drogues dans le corpus analysé.

\section{Typologie des lieux des drogues dans la presse quotidienne à trois échelons}

L'étude du corpus sous un angle plus spécifiquement géographique a permis de mettre en avant trois types de lieux associés aux drogues. On parle généralement d'entités nommées spatiales en textométrie. Ces différentes entités sont présentes aussi bien dans la $P Q N$ que la $P Q R$ mais avec des fréquences différentes. Pour relever ces différents lieux, nous avons parcouru manuellement la liste des formes présentes (figure 2). Les trois types de lieu relevés sont :

- des noms de villes (toponymes); 
- des lieux génériques;

- des lieux spécifiques.

Les villes de Marseille et Saint-Denis : des hauts lieux médiatiques de la drogue

Comme cela a déjà été évoqué, une partie des articles de la presse régionale concerne des faits se déroulant sur son aire de diffusion, de ce fait, les toponymes des villes appartenant à son territoire y sont particulièrement fréquents. De manière logique, Bordeaux apparait six fois plus dans les journaux diffusés dans le Sud-ouest que dans la presse nationale. Il est plus pertinent de se concentrer sur les autres villes mentionnées dans la presse, celles n'appartenant pas à nos terrains d'étude. Il s'avère que les toponymes Marseille et Saint-Denis ${ }^{13}$ sont particulièrement présents dans la presse nationale. La référence à ces deux toponymes dans la $\mathrm{PQN}$ contribue à la construction d'un imaginaire de la drogue, lié dans le cas de Marseille à l'histoire de la French connection qui continue à être entretenue à la fois dans les films et dans la presse (Marchant, 2012).

Tableau 4 - Fréquence d'apparition de certains toponymes dans les presses nationale et régionale

\begin{tabular}{|l|l|l|l|l|}
\hline \multirow{2}{*}{ Toponymes } & \multicolumn{2}{|l|}{$\begin{array}{l}\text { Occurrences } \\
\text { PQN }\end{array}$} & \multicolumn{2}{l|}{ Occurrences } \\
& VQR \\
\cline { 2 - 5 } & Val. Abs & $\%$ & Val. Abs & $\%$ \\
\hline Bordeaux & 357 & 0,6 & 1391 & 3,4 \\
\hline Marseille & 2195 & 3,9 & 382 & 0,9 \\
\hline Saint-Denis (ville ou dpt) & 660 & 1,2 & 171 & 0,4 \\
\hline Paris & 2749 & 4,9 & 685 & 1,66 \\
\hline
\end{tabular}

Outre une fréquence plus importante de ces toponymes dans la PQN (voir tab. 4), les classifications opérées avec la méthode Alceste décrite plus haut montrent une présence significative de ces formes dans les univers lexicaux de la violence et du trafic. Afin de mieux comprendre le contexte d'apparition de ces toponymes dans les articles, il est important de se référer au texte initial. Ainsi Marseille est très souvent le sujet d'articles relatant des homicides et des règlements de compte par armes à feu. Chaque année, la presse dénombre même le nombre de tués ou de fusillades dans cette ville.

Extraits de la PQN contenant « Marseille »

A la veille de la première visite du nouveau ministre de l'intérieur Bernard Cazeneuve, un homme de 25 ans a été tué par balles jeudi dans les quartiers nord de Marseille, sixième règlement de comptes depuis le début de l'année. (Libération, 24/04/2014)

Un trentenaire connu de la justice est victime de plusieurs balles dans la tête chemin des Escourtines dans le XIe arrondissement de Marseille. Deux autres faits similaires se sont produits en janvier dont une fusillade à la kalachnikov dans le quartier de la plaine. (20 Minutes, 07/02/2018)

Lorsque la ville de Saint-Denis ou son département sont présents dans la presse, c'est également comme haut lieu médiatique des drogues pour relayer des violences souvent 
meurtrières, mais ces dernières sont plus souvent évoquées dans le cadre du trafic de stupéfiants que ce n'est le cas pour la ville de Marseille, pour laquelle la description des circonstances de la tuerie semble se suffire à elle-même. La presse parle aussi de SaintDenis pour mettre en avant l'ampleur du trafic et l'économie qui en découle.

Extraits de la PQN contenant « Saint Denis »

La Seine-Saint-Denis, département où de nombreuses cités sensibles sont des plaques tournantes du trafic de drogue est annuellement le théâtre d'environ 80 règlements de comptes parfois mortels. En 2013, 30 homicides y ont été enregistrés dont une partie liée au trafic de drogue. (Libération, 17/06/2014)

Après la série de fusillades qui a marqué Saint-Ouen (Seine-Saint-Denis) sur fond de rivalités entre vendeurs de drogue, le ministre de l'intérieur Bernard Cazeneuve [...]). (La Croix, 15/05/2015)

On estime à 1 milliard d'euros le chiffre d'affaires de la drogue dans le département, l'équivalent de la moitié du budget du conseil départemental de Seine-Saint-Denis en 2015 (2,3 milliards d'euros). (Le Monde, 06/07/2016)

Il arrive aussi que les deux villes, ou leur département, soient présentes dans les mêmes articles. Lorsqu'il est question de criminalité en France ces lieux sont fréquemment cités en exemple en raison du nombre d'infraction qui s'y déroule mais aussi car les journalistes vont utiliser l'image que ces villes/départements véhiculent dans l'imaginaire collectif.

Extraits de la PQN contenant à la fois «Marseille» ou «Bouches du Rhône » et «Saint-Denis »

Malgré cet inquiétant palmarès, les Bouches du Rhône ne sont pas le département le plus criminogène de France : eu égard au taux d'infractions pour 1000 habitants, la Seine-Saint-Denis restait devant en 2012. (Le Monde, 07/09/2013)

Le banditisme sévit en Seine-Saint-Denis comme à Marseille. Il teste en permanence les résistances de l'Etat et il prospérera aussi longtemps que les voyous n'auront pas la certitude que la sanction tombera vite et fort. (Interview de B. Beschizza, Le Figaro, 07/08/2013)

Enfin, la forme Paris est très fréquente, en particulier dans la PQN. La capitale n'est pas considérée comme un haut-lieu des drogues mais comme celui du pouvoir politique. Ainsi, Paris est régulièrement utilisé comme figure rhétorique (synecdoque) pour évoquer les autorités locales ou nationales. Paris est aussi présente dans le corpus en raison de deux événements majeurs sur la période étudiée: les attentats de novembre 2015 et l'installation de la salle de consommation à moindre risque dans le 10 ème arrondissement. Des articles sur les attentats sont dans le corpus en raison des mots-clés choisis car ils évoquent le fait que les auteurs n'étaient pas «drogués » et que leur logeur était un trafiquant. Par ailleurs, les salles de consommation à moindre risque de Paris et Strasbourg ouvertes depuis l'automne 2016 ont donné lieu à des controverses politiques rendant leur implantation locale complexes. Il en était prévu une à Bordeaux qui n'existe finalement pas, mais dont la presse locale s'est aussi fait l'écho.

\section{Des lieux génériques : quartier et cité, des espaces stigmatisés}

Une fois les faits localisés dans une ville, il est fréquent que les journalistes précisent la localisation à un échelon inférieur. C'est là qu'apparait le vocabulaire des lieux génériques de la ville tels que « rue $\mathrm{X}$ », «place $\mathrm{Y}$ », «parking $\mathrm{Z}$ » ou « centre-ville». Ces noms communs de lieux, associés à leur nom propre permettent de donner une information plus précise mais parfois aussi de stigmatiser certains espaces de la ville. 
C'est ainsi que deux types de lieux, eux-mêmes parfois connotés, sont très présents en particulier dans la presse nationale. Il s'agit de quartier et cité. Ces termes peuvent être utilisés dans un sens neutre et, dans le cas du quartier par exemple, simplement désigner et localiser une portion d'espace de la ville. Cependant, la plupart du temps, quartier comme cité sont utilisés en référence aux grands ensembles de banlieue, où le trafic et la violence sont particulièrement médiatisés dans la PQN qui traite nombre de ces sujets et les associe notamment aux villes de Marseille et Saint-Denis.

Tableau 5 - Fréquence d'apparition de « quartier » et « cité » dans la PQN et la PQR

\begin{tabular}{|l|l|l|l|l|}
\hline \multirow{2}{*}{ Lieu } & \multicolumn{2}{|l|}{ Occurrences PQN } & \multicolumn{2}{l|}{ Occurrences PQR } \\
\cline { 2 - 5 } & Val. Abs. & $\%$ & Val. Abs. & $\%$ \\
\hline Quartier & 2494 & 4,4 & 1060 & 2,6 \\
\hline Cité & 1575 & 2,8 & 408 & 1 \\
\hline
\end{tabular}

Aujourd'hui en France, une partie de la population est associée à ce type de lieu et si les stigmates et les catégories sociales évoluent dans le temps, les médias s'en font toujours l'écho tel que le montre P.-O. Dupuy: «Les jeunes de banlieue issus de l'immigration incarnent, en tant que catégorie sociale menaçante, les blousons noirs d'hier (Boyer \& Lochard, 1998) [...]. A chaque nouveau phénomène d'être comparé, réactualisé, catégorisé et chargé de connotations positives ou négatives (Lochard, 2003). Les catégories sociales sont des outils linguistiques, évaluatifs et idéologiques qui viennent construire la nature des groupes (Augoustinos et Quinn, 2003).» (Dupuy, 2008). Ainsi, la presse, par son discours médiatisé, participe à la construction et à la diffusion de catégories sociales et spatiales plus ou moins caricaturales et stigmatisées.

Extraits de la PQN contenant « quartier » ou « cité »

La police marseillaise a annoncé le démantèlement de plusieurs réseaux de trafic de drogue et la saisie de nombreuses armes et d'argent sale dans les cités. (Le Monde, 08/01/2014)

Dans ces quartiers à lourde précarité le taux de chômage des 15-24 ans grimpe jusqu'à 50\%. La vente de stupéfiants s'appuie sur ce réservoir inépuisable de jeunes garçons qui souvent cumulent rupture scolaire, fragilité familiale et absence de perspectives d'avenir. (La Croix, 13/09/2016)

\section{Des lieux spécifiques : de la répression à l'accompagnement des usages}

Une dernière catégorie de lieux spécifiques, relevant surtout de l'échelle micro, est présente dans le corpus. La liste des formes nous permet de relever des lieux appartenant au champ du judiciaire. En effet, la criminalisation et la judiciarisation des drogues sont des thèmes récurrents dans la presse, d'où cette catégorie qui regroupe des lieux tels que : gendarmerie, à la barre, tribunal ou prison. Ces lieux reflètent les cheminements de l'usager ou du dealer dans leur parcours judiciaire de l'arrestation à l'emprisonnement en passant par le jugement. Cette géographie du judiciaire fait partie de la catégorie plus large regroupant tout le vocabulaire appartenant à ce champ lexical ainsi que le montrent le nuage de mots et la classification présentés précédemment dans l'article. 

de la France, une autre catégorie de lieu spécifique apparait, celle du trafic frontalier. Des mots-lieux tels que douane, autoroute, frontière ou péage montrent une géographie du trafic frontalier plus présente dans la presse régionale. Cette géographie pourrait se retrouver dans la presse de n'importe quelle région frontalière confrontée à cette problématique. Dans le cas de la presse étudiée, ces termes sont associés à des toponymes tels que Espagne et Maroc ou des localités du Pays Basque dont la fréquence est plus importante que dans la PQN. Comme pour les lieux du judiciaire, cette géographie du trafic frontalier appartient à la catégorie plus vaste du trafic, également mise en avant grâce à la classification et dans laquelle se retrouvent d'autres termes appartenant à ce champ lexical.

Extrait de la $\mathrm{PQR}$ contenant du vocabulaire géographique spécifique au trafic

Les douaniers bordelais affectionnent les aires autoroutières car ils savent que Bordeaux constitue un passage quasi obligé pour les convois chargés de produits stupéfiants remontant du Maroc via l'Espagne que ce soit à bord de voitures ou de poids lourds. (Sud-Ouest, 09/12/2017)

Enfin, comme nous l'évoquions précédemment, durant cette période 2013-2018, un certain nombre d'article fait référence aux salles de consommation à moindre risque, dont l'acronyme est SCMR mais qui sont plus souvent appelées "salles de shoot» dans la presse. L'expression apparait à 370 reprises dans la PQN et 115 fois dans la PQR. Il est également possible d'y voir la tendance sensationnaliste de certains titres si l'on prend en considération la sur-utilisation de cette expression, fortement connotée, en comparaison du faible emploi de termes moins connotés tels que salle d'injection ou salle de consommation (à moindre risque).

différents lieux cités sont le reflet de la politique française en matière de drogue mais également de la «tyrannie» de l'opinion interrogée dans des sondages fréquemment afin de justifier les décisions politiques (Jauffret-Roustide et al., 2013). Dans ces sondages, les Français sont interrogés sur les politiques des drogues (salles de consommation à moindre risque entre autres) sans être réellement informés des objectifs de ces salles.

51 La loi française criminalise la vente et l'usage, d'une part, d'où la présence des lieux de trafic et de traitement judiciaire dans la presse. D'autre part, la politique des drogues a aussi un versant de prévention et d'accès aux soins avec des lieux dédiés, dont les SCMR qui apparaissent également dans le corpus. Ce type de lieu de soin et de réduction des risques dans la presse a d'ailleurs fait l'objet d'une étude récente, mettant en évidence la prédominance de la parole politique et l'invisibilité de la parole des usagers de drogues (Jauffret-Roustide et Caillbault, 2018).

\section{Conclusion}

L'étude qui sous-tend cet article nous a permis de valider des hypothèses du point de vue méthodologique et des résultats scientifiques. Nous avons pu montrer, si tant est que cela soit nécessaire, de la richesse qu'apporte une approche pluridisciplinaire tant pour les savoirs que pour les méthodes. Concernant la méthodologie employée, nous avons pu confirmer l'intérêt de prendre en compte la presse parmi les sources d'information, aussi bien pour saisir un point de vue non négligeable, celui reflétant et/ ou influençant l'opinion publique et les politiques, que pour saisir une géographie des 
drogues à différents niveaux. Pour cela, le choix de la textométrie via l'utilisation d'un logiciel d'analyse textuelle qui permet d'automatiser une partie des analyses s'est révélé pertinent pour étudier un corpus de cette taille. Cette méthode mixte a de plus l'avantage de permettre une double approche: quantitative en réalisant des statistiques sur des grands nombres, et qualitative en permettant de retourner au contexte d'énonciation à travers les segments de texte. Cette méthode nous a permis de mettre en avant des résultats concernant la presse et la géographie des drogues.

Nous avons ainsi pu constater que la presse, même celle qualifiée de "qualité » (Merril, 2000) a une tendance au sensationnalisme (Sedel, 2009), en particulier la presse nationale, ce qui participe à la stigmatisation de certains espaces tels que les quartiers populaires (Derville, 1997). Ces faits divers relayant des activités criminelles et/ou la judiciarisation des faits liés à la drogue reflètent le contexte politique prohibitionniste de la France en matière de drogues qui est en décalage avec un mouvement international de légalisation ou de décriminalisation dans les pays à niveaux de revenus comparables (Obradovic, 2015; Jauffret-Roustide et Granier, 2017). L'analyse de la presse à deux échelons de diffusion s'est révélée pertinente pour mettre en avant des nuances tant thématiques que géographiques. Ainsi l'aire de diffusion de la presse influe notamment sur son contenu géographique, ce que nous avons pu montrer avec l'exemple de la géographie du trafic frontalier France/Espagne dans la presse du sudouest. Enfin, cette étude nous a permis de réaliser une typologie des lieux des drogues faite de trois catégories: des noms de villes parmi lesquels revenaient souvent celles évoquant un imaginaire violent (Marseille, Saint Denis), des lieux génériques dont certains peuvent aussi renvoyer à cet imaginaire stigmatisant (cité, banlieue) et enfin des lieux spécifiques évoquant notamment le champ du judiciaire renvoyant au contexte prohibitionniste français (tribunal, prison).

Enfin, les résultats de cette étude sont le produit de choix méthodologiques faits à chaque étape (Pincemin, 2011), selon ce qui nous paraissait le plus pertinent compte tenu de nos connaissances en matière de textométrie et des capacités de traitement du matériel informatique. De plus, le corpus est d'une richesse telle que nous aurions, par exemple, également pu d'avantage analyser le contexte en étudiant le rôle des orientations politiques des journaux, l'évolution de la thématique au fil du temps ou encore questionner la place physique de la drogue (en prenant en compte les rubriques ou les pages où sont ces articles). Ces limites sont autant de pistes complémentaires à explorer.

\section{BIBLIOGRAPHIE}

Albert P., 2016. La PQR et la politique. La revue européenne des médias et du numérique. $\mathrm{n}^{\circ} 37$.

Ash J., Kitchin R., Leszczynski A., 2016. Digital turn, digital geographies?. Progress in Human Geography, vol. ${ }^{\circ} 42, \mathrm{n}^{\circ} 1$, p. $25-43$. 
Ballarini L., 2008. Presse locale, un média de diversion. Réseaux, n 148-149, p. 405-426. URL: https://www.cairn.info/revue-reseaux1-2008-2-page-405.html

Beauguitte L., Grasland C.,Severo M., 2016. Espaces géographiques et représentations médiatiques. L'Espace géographique, tome 45, p. 1-4.

Beck F., Peretti-Watel P., 2001. L'héroïne entre répression et réduction des risques : comment sont perçues les politiques publiques? Sociétés contemporaines [En ligne sur Cairn], n 41-42, p. 133-158. URL: https://www.cairn.info/revue-societes-contemporaines-2001-1-page-133.htm

Beck F., Richard J.-B., Guignard R., et al., 2015. Les niveaux d'usage des drogues en France en 2014. Tendances, $\mathrm{n}^{\circ}$ 99, OFDT, $8 \mathrm{p}$.

Bregman D., 1989. La fonction d'agenda : une problématique en devenir. Hermès, La Revue [En ligne], nº 4, p. 191-202. URL: http://hdl.handle.net/2042/15409 - DOI: https://doi.org/ 10.4267/2042/15409https://doi.org/10.4267/2042/15409

Brissot A., et al., 2019. Drogues : perceptions des produits, des politiques publiques et des usagers. Tendances, $\mathrm{n}^{\circ} 131$, OFDT, $8 \mathrm{p}$.

Burgess J., 1990. The production and consumption of environmental meanings in the mass media: A research agenda for the 1990s. Transactions of the Institute of British Geographers, vol. 15, $n^{\circ} 2$, p. 139-161.

Burgess J., Gold J.R. (ed.), 1985. Geography, the media and popular culture. New York, St. Martin's Press, $272 \mathrm{p}$.

Cabré T., 2008. Constituer un corpus de textes de spécialité. Les cahiers du CIEL, p. 37-56.

Chouvy P.-A., 2019. Les frontières, fronts inefficaces de la lutte contre le trafic international de drogue. L'Espace Politique [En ligne], $\mathrm{n}^{\circ}$ 38. URL http://journals.openedition.org/espacepolitique/ 6496 - DOI: https://doi.org/10.4000/espacepolitique.6496

Chouvy P.-A., 2017. La géographie à l'épreuve de la drogue. Dossier d'habilitation à diriger des recherches (HDR), vol. 1, $144 \mathrm{p}$.

Dagiral E., Parasie S., 2010. Presse en ligne : où en est la recherche ? Réseaux, n 160-161, p. 13-42.

Dany L. Apostolidis T., 2002. L'étude des représentations sociales de la drogue et du cannabis : un enjeu pour la prévention. Santé Publique, vol. 14 n 4, p. 335-344.

Debarbieux B., 1998. Les problématiques de l'image et de la représentation en géographie. In Bailly A. (coord.), Les concepts de la géographie humaine. Paris, Armand Colin, p. 199-211.

Delporte C., Blandin C., Robinet F., 2016. Chapitre 10. Le monde de la presse écrite : un monde en crise ? In Delporte C., Blandin C., Robinet F. (dir.), Histoire de la presse en France : XX ${ }^{e}-X^{2} I^{e}$ siècles. Paris, Armand Colin, p. 242-262.

Derville G., 1997. La stigmatisation des « jeunes de banlieue ». Communication et langages, $\mathrm{n}^{\circ} 113$, p. 104-117.

Di Méo, G., 2008. Une géographie sociale entre représentations et action. Montagnes méditerranéennes et développement territorial. Institut de géographie alpine. p. 13-21.

Dupuy P.-O., 2008. L'interaction politique vue comme un processus de construction identitaire : le cas des présidentielles 2007 : analyse du débat de l'entre-deux-tours et des questions de citoyens. Thèse de doctorat en Sciences de l'information et de la communication. Université Paul Sabatier, Toulouse 3. 
Ehrmann M., 2008. Les Entitées Nommées, de la linguistique au TAL: Statut théorique et méthodes de désambiguïsation. Thèse de doctorat en linguistique théorique, descriptive et automatique. Université Paris Diderot.

Fallery B., Rhodain. F., 2007. Quatre approches pour l'analyse de données textuelles: Lexicale, linguistique, cognitive, thématique. XVI ${ }^{\text {ème }}$ Conférence de l'Association Internationale de Management Stratégique AIMS, Montréal, Canada. AIMS, p. 1-16, URL: https://hal.archivesouvertes.fr/hal-00821448/document

Frémont A., 1976. La région, espace vécu. Paris, PUF, 223 p.

Fredrickson A., Gibson A., Lancaster K., Nathan, S., 2019. “Devil's lure took all I had”: Moral panic and the discursive construction of crystal methamphetamine in Australian news media. Contemporary Drug Problems, vol. 46, n 1, p. 105-121.

Guérin-Pace F., 1997. La statistique textuelle. Un outil exploratoire en sciences sociales. Population, $52^{\mathrm{e}}$ année, $\mathrm{n}^{\circ}$ 4, p. 865-887. URL: www.persee.fr/doc/ pop_0032-4663_1997_num_52_4_6471

Guérin-Pace F., Saint-Julien T., 2012. Une analyse lexicale des titres et mots-clés de 1972 à 2010. L'Espace géographique, $\mathrm{n}^{\circ}$ 41, p. 4-30. URL: https://www.cairn.info/revue-espacegeographique-2012-1-page-4.htm - DOI: https://doi.org/10.3917/eg.411.0004

Jauffret-Roustide M., 2009. Un regard sociologique sur « les drogues : décrire la complexité des usages et rendre compte des contextes sociaux. La revue lacanienne [En ligne sur Cairn], $\mathrm{n}^{\circ} 5, \mathrm{p}$. 109-118. URL: https://www.cairn.info/revue-la-revue-lacanienne-2009-3-page-109.htm

Jauffret-Roustide M., Pedrono G., Beltzer N., 2013. Supervised consumption rooms: the French paradox. International Journal of Drug Policy, vol. 24, nº 6, p. 628-30.

Jauffret-Roustide M., Granier J.M., 2017. Repenser la politique des drogues. Esprit, nº 431, p. 40-54. Jauffret-Roustide M., Cailbault I., 2018. Drug consumption rooms: Comparing times, spaces and actors in issues of social acceptability in French public debat. International journal of drug policy, $\mathrm{n}^{\circ}$ 58, p. 208-217.

Lancaster K., et al., 2011. Illicit drugs and the media: Models of media effects for use in drug policy research. Drug and Alcohol Review, vol. 30, n 4, p. 397-402.

Leteinturier C., 2021. Fait divers. In Encyclopcedia Universalis.

Le Nézet O., et al., 2019. Usages de substances illicites. Workbook 3. In Rapport national à l'EMCDDA, OFDT.

Marpsat M., 2010. La méthode Alceste. Sociologie [En ligne], vol. 1, nº 1. URL: http:// journals.openedition.org/sociologie/312

Mains S., Cupples J., Lukinbeal C. (ed.), 2015. Mediated Geographies and Geographies of Media. Dordrecht, Springer, $470 \mathrm{p}$.

Marchant, A., 2012. La French Connection, entre mythes et réalités. Vingtième Siècle, Revue d'histoire, $\mathrm{n}^{\circ}$ 115, p. 89-102.

Merril J.C., 2000. Les quotidiens de référence dans le monde. Les Cahiers du journalisme, ${ }^{\circ} 7,14 \mathrm{p}$. Obradovic I., 2015. Trente ans de réponse pénale à l'usage de stupéfiants. Tendances, $\mathrm{n}^{\circ} 103$, OFDT, $6 \mathrm{p}$. 
Pincemin B., 2011. Sémantique interprétative et textométrie. Version abrégée. Corpus [En ligne], $\mathrm{n}^{\circ}$ 10. URL: http://journals.openedition.org/corpus/212 - DOI: https://doi.org/10.4000/corpus. 2121

Reinert M., 1990. Alceste, une méthodologie d'analyse des données textuelles et une application : Aurélia de G. de Nerval. Bulletin de méthodologie sociologique, n² 26, p. 24-54.

Reinert M., 1999. Quelques interrogations à propos de l'"objet" d'une analyse de discours de type statistique et de la réponse "Alceste". Langage \& Société, n 90, p. 57-79.

Resch M., Lavie E., Arnaud-Fassetta G., 2020. "La Voulzie à Paris” : étude des oppositions à la dérivation des sources de la Voulzie à partir des discours de la presse écrite (1885-1929). Cybergeo: European Journal of Geography [En ligne], document 960. URL: http://journals.openedition.org/ cybergeo/35713 - DOI: https://doi.org/10.4000/cybergeo.35713

Rouré H., Reinert M., 1993. Analyse d'un entretien à l'aide d'une méthode d'analyse lexicale. Journées internationales d'analyse des données textuelles. Paris, ENST.

Sedel J., 2009. Les médias \& la banlieue. Lormont, Le Bord de l'eau, 300 p.

Venturini T., 2012. Great Expectations: Méthodes Quali-Quantitative Et Analyse Des Réseaux Sociaux. In Fourmentraux, J.P. (dir.), L'Ère Post-Media. Humanités digitales et Cultures numériques. Paris, Hermann, p. 39-51.

\section{NOTES}

1. DRUSEC (Drug and Urban Securities) est un projet franco-allemand ANR-BMBF (2017-2021)

2. https://geomedia.hypotheses.org/

3. https://www.ofdt.fr/enquetes-et-dispositifs/eropp/

4. Voir notamment les travaux de Pierre-Arnaud Chouvy $(2017,2019)$.

5. http://www.iramuteq.org/

6. Initialement ALCESTE était l'acronyme de «Analyse Lexicale par Contexte d'un Ensemble de Segments de Texte», depuis Reinert utilise «Analyse des Lexèmes Cooccurrents dans un Ensemble de Segmentations du Texte Étudié ».

7. http://www.iramuteq.org/

8. https://www.r-project.org/

9. Méthode qui consiste à chaque étape à diviser la plus grande classe en deux

10. En phase expérimentale pour 2 ans à compter d'octobre 2021, auprès de 3000 patients. (https://www.service-public.fr/particuliers/actualites/A14362).

11. Voir classe 2 « violence/crime » à droite sur la figure 3 (classification $P Q N)$.

12. Voir classe 4 « violence/délit » à gauche sur la figure 4 (classification $P Q R$ ).

13. La forme « Saint-Denis » regroupe les occurrences concernant la ville et le département.

\section{RÉSUMÉS}

Les médias sont devenus des objets d'étude et des outils de plus en plus utilisés en sciences sociales. Les discours véhiculés par ces derniers participent à façonner les représentations 
sociales, les représentations spatiales et les imaginaires géographiques. Cet article a une double ambition : il s'agit, d'une part, de présenter un outil et une méthode d'analyse de la presse en des termes accessibles à des non spécialistes de l'analyse textuelle et, d'autre part, de présenter les résultats obtenus grâce à cette méthode à la question : quels traitements thématiques et spatiaux des drogues dans la presse quotidienne française ? Nous avons ainsi cherché à savoir quels sont les angles thématiques choisis par les journaux et quelle(s) géographie(s) des drogues est repérable à partir de ce traitement médiatique. Ces deux entrées nous renseignent sur le contexte politique et sur les représentations véhiculées par et autour de cet objet d'étude sensible que sont les drogues.

The media have become objects of study and tools increasingly used by the humanities and social sciences. The discourses conveyed by the latter participate in shaping social representations, spatial representations and geographical imaginaries. This article has a double ambition: on the one hand, to present a tool and a method of analysis of the press in terms accessible to nonspecialists of textual analysis and, on the other hand, to present the results obtained thanks to this method to the question: which thematic and spatial treatments of drugs in the French daily press? We thus sought to know what thematic were chosen by the newspapers and what geography(ies) of drugs could be identified from this media treatment. These two entries provide information on the political context and on the representations conveyed by and around this sensitive object of study.

\section{INDEX}

Mots-clés : drogue, textométrie, analyse de la presse, représentation socio-spatiale

Keywords : drug, textometry, press analysis, socio-spatial representation

Thèmes : Sur le Métier

\section{AUTEURS}

\section{CÉCILIA COMELLI}

Cécilia Comelli, Cecilia.comelli01@gmail.com, est chercheure associée de l'UMR 5319 Passages.

Elle a récemment publié :

- Comelli, C., 2021. L'insertion délicate des dispositifs d'accompagnement des usagers de drogue précaires dans le tissu urbain bordelais. Espace populations sociétés [En ligne], $n^{\circ} 2-3 \mid 2021$. URL: http://journals.openedition.org/eps/11749 - DOI: https://doi.org/10.4000/eps.11749

- Comelli C., 2019. Ambivalence et complexité des nuits urbaines contemporaines : le cas de Bordeaux. Bollettino della Società Geografica Italiana, $n^{\circ}$ 14, p. 85-97.

- Mallet S., Comelli C., 2017. Politiques d'éclairage public et transformations des espaces urbains : une approche critique. Cybergeo: Revue européenne de géographie / European journal of geography [En ligne], document 833. URL: http://journals.openedition.org/cybergeo/28796 - DOI: https:// doi.org/10.4000/cybergeo.28796

\section{GRÉGOIRE LE CAMPION}

Grégoire Le Campion, gregoire.lecampion@cnrs.fr, est ingénieur d'étude à l'UMR 5319 Passages. Il a récemment publié :

- Guyot S., Le Campion G., Pissoat O., 2020. Diversité et enjeux territoriaux de la mise en art des espaces périphériques dans le monde. Cybergeo: Revue européenne de géographie / European journal of 
geography [En ligne], document 962. URL: http://journals.openedition.org/cybergeo/35837 - DOI: https://doi.org/10.4000/cybergeo.35837

- Mondo, M., Noucher, M., Le Campion, G., et al., 2020. Critical approach of digital footprints to study spatial practices of urban tourist areas: a case study of Instagram data in Biarritz (France). WIT Transactions on Ecology and the Environment, p. 319-333. URL: https://hal.archives-ouvertes.fr/ hal-03114956/document

- Le Campion G. et al., 2019. 30 ans d'articles dans Mappemonde. Une exploration par les mots-clés et les résumés. M@ppemonde [En ligne], n 127. URL: http://journals.openedition.org/ mappemonde/2494 - DOI: https://doi.org/10.4000/mappemonde.2494

\section{MARIE JAUFFRET-ROUSTIDE}

Marie Jauffret-Roustide, Marie.JAUFFRETROUSTIDE@santepubliquefrance.fr, est chargée de recherche Inserm au Centre d'Étude des Mouvements Sociaux (Inserm U1276/CNRS UMR8044/ EHESS) ; Affiliate Scientist at the British Columbia Center For Substance Use (BCCSU), Vancouver, Canada ; and Senior Research Fellow at the Bady Center on Law and Social Policy, Buffalo, NY, USA. Elle a récemment publié :

- Jauffret-Roustide M., Caillbault I., 2018. Drug consumption rooms: comparing times, spaces and actors in issues of social acceptability in French public debate. International Journal of Drug Policy, vol.56, p. 208-217. 56:208-217. DOI: https://doi.org/10.1016/j.drugpo.2018.04.014

- Jauffret-Roustide M., Granier J.M., 2017. Repenser la politique des drogues. Esprit, $n^{\circ} 431$, p. $40-54$.

- Jauffret-Roustide M., 2014. Les inégalités sociales dans le champ des addictions. Tribunes de la santé, Presses de Sciences Po, n²4, p. 61-68. 